\title{
Estimation of disability weight for paragonimiasis: a systematic analysis
}

Yun Feng ${ }^{1,2,3}$, Thomas Fürst ${ }^{4,5}$, Lu Liu' ${ }^{1,2,3}$ and Guo-Jing Yang ${ }^{1,2,3,4,5^{*}}$

\begin{abstract}
Background: Paragonimiasis, caused by helminths of the genus Paragonimus spp., is a neglected tropical disease. Human suffering from paragonimiasis is often misunderstood and its quantification by the disability weight of the disability-adjusted life years largely varies in different global burden of disease (GBD) estimates. This paper is to systematically review clinical paragonimiasis cases and requantify the disability weight of human paragonimiasis.

Methods: A systematic analysis was conducted using articles from the following databases: PubMed, Institute for Scientific Information Web of Science, China National Knowledge Infrastructure, the Chinese scientific journal databases Wanfang Data and CQVIP, Africa Journal Online, and the System for Information on Grey Literature in Europe. Search terms were the combination of "paragonim*" with "clinical" or "infection". Only articles fulfilling the following conditions were recruited for this study: the occurrence of clinical signs and symptoms of paragonimiasis in human beings were reported; diagnosis was confirmed; no comorbidities were reported; the reviewed clinical cases or epidemiological findings were not already included in any other articles. The information and frequencies of paragonimiasis outcomes from included articles using predefined data fields were extracted two times by two separate individuals. Outcome disability weights were selected mainly from the GBD 2004 and GBD 2013 datasets. Frequencies and disability weights of paragonimiasis outcomes were modelled into a decision tree using the additive approach and multiplicative approach, respectively. Monte Carlo simulations were run 5000 times for an uncertainty analysis.

Results: The disability weight estimates of paragonimiasis were simulated with 5302 clinical cases from 80 general articles. The overall disability weight was estimated at 0.1927 (median 0.1956) with a 95\% uncertainty interval (UI) of $0.1632-0.2378$ using the additive approach, and 0.1791 (median 0.1816 ) with a $95 \%$ UI of $0.1530-0.2182$ using the multiplicative approach. The simulated disability weights of Paragonimus westermani cases were higher than that of P. skrjabini cases. Lung outcomes and headache were the top two contributors to disability weight for both species.

Conclusions: The use of paragonimiasis disability weight needs to be reconsidered with regard to availability of morbidity data and species variation. Calculating the disease burden of paragonimiasis requires further modification and thus has considerable implications for public health prioritization in research, monitoring, and control.
\end{abstract}

Keywords: Paragonimiasis, Disability weight, Disease burden, Disability-adjusted life years

\footnotetext{
* Correspondence: guojingyang@hotmail.com

'Jiangsu Institute of Parasitic Diseases, 117 Meiyuan Yangxiang, Wuxi City,

Jiangsu Province 214064, People's Republic of China

${ }^{2}$ Key Laboratory of National Health and Family Planning Commission on

Parasitic Disease Control and Prevention, Wuxi, People's Republic of China

Full list of author information is available at the end of the article
}

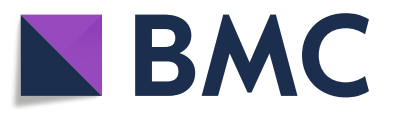

(c) The Author(s). 2018 Open Access This article is distributed under the terms of the Creative Commons Attribution 4.0 International License (http://creativecommons.org/licenses/by/4.0/), which permits unrestricted use, distribution, and reproduction in any medium, provided you give appropriate credit to the original author(s) and the source, provide a link to the Creative Commons license, and indicate if changes were made. The Creative Commons Public Domain Dedication waiver (http://creativecommons.org/publicdomain/zero/1.0/) applies to the data made available in this article, unless otherwise stated. 


\section{Multilingual Abstracts}

Please see Additional file 1 for translations of the abstract into the five official languages of the United Nations.

\section{Background}

Paragonimiasis is a neglected tropical disease (NTD) [1] caused by helminths of the genus Paragonimus spp., whose eggs hatch in freshwater and yield motile miracidia. The miracidia further develop into motile microcercous cercariae in freshwater snails and later grow into metacercariae in crustaceans like crab or crayfish. Ingestion of contaminated, undercooked freshwater crabs or crayfish leads to paragonimiasis [2].

Paragonimiasis is not usually considered a national or regional public health priority, but due to its wide distribution and severe health consequences in endemic regions, the disease should not be neglected. Paragonimiasis is endemic in parts of Africa, North and South America, and particularly in Southeast and East Asian countries such as China, Japan, and the Philippines [3, 4]. The prevalence of paragonimiasis in northeastern India can reach as high as $51.7 \%$ in children aged under 15 years and $18.7 \%$ in adults [5]. The disease can also occur in non-endemic areas; for instance, in returning travellers or through infections caused by internationally traded and contaminated raw freshwater crab or crayfish $[2,6]$. With the gradually increasing aquaculture production of freshwater crustacea worldwide [7], the endemicity and re-emergence of paragonimiasis deserve more attention [8].

Paragonimiasis causes complex symptoms in multiple organs. Chest symptoms are its most remarkable clinical features, as it is mainly in the lung parenchyma that adult worms exist in pairs encapsulated in fibrous cysts [2, 9]. For some Paragonimus species such as P. skrjabini, only a few of the parasites develop into adults in the lungs, while most others migrate to various organs including muscles, subcutaneous tissue, brain, liver, and so on, at the juvenile stage $[9,10]$. Such ectopic infections can cause a variety of signs and symptoms including subcutaneous mass, headache, epilepsy, diarrhoea $[9,11,12]$.

Probably due to its status as a NTD, suffering from paragonimiasis is often misunderstood and its quantification in the disability weight (DW) of disability-adjusted life years (DALYs) largely varies in global burden of disease (GBD) estimates. Fürst et al. [3] estimated the disease burden of paragonimiasis based on existing DWs pertaining to most similar outcomes, which were chronic outcomes of lower respiratory infection for heavy pleuropulmonary infections

Table 1 DWs used in disease burden estimation

\begin{tabular}{|c|c|c|c|c|}
\hline Disease burden estimation & Outcome & Proxy health state & Proxy DW & Reference \\
\hline \multirow[t]{7}{*}{ GBD 2016} & Asymptomatic paragonimiasis & $\mathrm{N} / \mathrm{A}$ & $\mathrm{N} / \mathrm{A}$ & {$[18]$} \\
\hline & Heavy paragonimiasis & TB, not HIV infected & $0.333(0.224-0.454)$ & \\
\hline & $\begin{array}{l}\text { Mild paragonimiasis due to } \\
\text { foodborne trematodiases }\end{array}$ & $\begin{array}{l}\text { COPD and other chronic } \\
\text { respiratory problem, mild }\end{array}$ & $0.019(0.011-0.033)$ & \\
\hline & $\begin{array}{l}\text { Moderate paragonimiasis } \\
\text { due to foodborne trematodiases }\end{array}$ & $\begin{array}{l}\text { COPD and other chronic } \\
\text { respiratory problem, moderate }\end{array}$ & $0.225(0.153-0.31)$ & \\
\hline & $\begin{array}{l}\text { Severe paragonimiasis } \\
\text { due to foodborne trematodiases }\end{array}$ & $\begin{array}{l}\text { COPD and other chronic } \\
\text { respiratory problem, severe }\end{array}$ & $0.408(0.273-0.556)$ & \\
\hline & \multirow[t]{2}{*}{ Cerebral paragonimiasis } & $\begin{array}{l}\text { Epilepsy, less severe (seizure } \\
<1 \text { per month) }\end{array}$ & $0.263(0.173-0.367)$ & \\
\hline & & $\begin{array}{l}\text { Epilepsy, severe (seizure } \\
\geq 1 \text { per month) }\end{array}$ & $0.552(0.375-0.710)$ & \\
\hline \multirow[t]{4}{*}{ GBD 2013} & Asymptomatic paragonimiasis & N/A & N/A & {$[16,17]$} \\
\hline & Heavy paragonimiasis & TB, not HIV infected & $0.333(0.224-0.454)$ & \\
\hline & \multirow[t]{2}{*}{ Cerebral paragonimiasis } & $\begin{array}{l}\text { Epilepsy, less severe (seizure } \\
<1 \text { per month) }\end{array}$ & $0.263(0.173-0.367)$ & \\
\hline & & $\begin{array}{l}\text { Epilepsy, severe (seizure } \\
\geq 1 \text { per month) }\end{array}$ & $0.552(0.375-0.710)$ & \\
\hline WHO FERG & Paragonimiasis & Uncontrolled asthma & $0.132(0.087-0.190)$ & {$[15,21,22]$} \\
\hline \multirow[t]{2}{*}{ GBD 2010} & Heavy paragonimiasis & Abdominopelvic problem: moderate & $0.123(0.083-0.176)$ & {$[14,15,23,24]$} \\
\hline & Cerebral paragonimiasis & Abdominopelvic problem: moderate & $0.123(0.083-0.176)$ & \\
\hline \multirow[t]{2}{*}{ Fürst et al. } & Heavy paragonimiasis & Lower respiratory infection & 0.099 & {$[3]$} \\
\hline & Cerebral paragonimiasis & $\begin{array}{l}\text { Seizure disorders due } \\
\text { to meningitis }\end{array}$ & 0.100 & \\
\hline
\end{tabular}


and seizure disorders due to meningitis for cerebral paragonimiasis (see also [13]). The GBD 2010 study used the newly developed DW of the health status "moderate abdominal pelvic problem" for the outcomes of both heavy pleuropulmonary and cerebral paragonimiasis $[14,15]$. As it is clinically incorrect, and signs and symptoms of pleuropulmonary and cerebral paragonimiasis do not really match those of moderate abdominopelvic problems, the GBD 2013 used the DWs for tuberculosis (TB) and epilepsy as proxies [16, 17]. The GBD 2016 continued with similar usage, but an extra proxy DWs of chronic obstructive pulmonary disease (COPD) with different severity levels were also used [18] (see Table 1). In fact, cases of pleuropulmonary paragonimiasis often present with asthma-, bronchitis-, and TB-like signs and symptoms, but their general health state is usually better than that of TB patients $[9,11,19]$. Realizing this and relying on the then most recent DWs from the GBD 2010 study, the World Health Organization's Foodborne Disease Burden Epidemiology Reference Group (WHO FERG) applied the DW for uncontrolled asthma instead of the more than two times higher DW for TB as a proxy for pleuropulmonary paragonimiasis in their latest estimates $[15,20,21]$.

The substantial differences in DWs for paragonimiasis translate into substantial differences in the thus far published GBD estimates and thereby limit the usefulness of all these estimates $[3,20,22-25]$. Also of note is that all the aforementioned estimates considered only pleuropulmonary and cerebral paragonimiasis, however, additional outcomes that were associated with paragonimiasis, such as pericarditis due to heart involvement, for instance [12], should also be considered.

Due to all these factors, we systematically reviewed clinical paragonimiasis cases and applied decision tree modelling approaches to requantify the DW for human paragonimiasis.

\section{Methods}

\section{Search strategy and selection criteria}

The systematic literature search included the databases PubMed, Institute for Scientific Information (ISI) Web of Science, China National Knowledge Infrastructure (CNKI), the Chinese scientific journal databases Wanfang Data and CQVIP, Africa Journal Online (AJOL), and the System for Information on Grey Literature in Europe (SIGLE). Search terms were the combination of "paragonim"" with "clinical" or "infection". Articles were reviewed by title and abstract, and subsequently full text.

The inclusion criteria were: (i) the articles described the occurrence of clinical signs and symptoms of paragonimiasis in human beings; (ii) the articles reported on paragonimiasis only (i.e. articles reporting obvious comorbidities were excluded); (iii) the diagnosis of paragonimiasis cases was confirmed by parasitological, serological, or histological evidence; and (iv) the article reviewed clinical cases or epidemiological findings that were not already included in other articles. The year of the study and the year of publication were irrelevant in the inclusion/exclusion of articles. Articles in languages other than English or Chinese, or without full text available to authors, were excluded. Articles that selectively reported on a specific case group such as cerebral paragonimiasis were also excluded. The study selection process is shown in Fig. 1.

\section{Data extraction and processing}

Two authors extracted the information from included articles. Information was extracted according to the following predefined data fields: case recruitment method (case reviews/surveys); region (country, province); Paragonimus species; and outcomes reported and their relevant occurrences.

The reported outcomes covered the following items: lung outcome (chest X-ray/ computed tomography shadow with symptoms such as fever or cough); pleural outcome (pleural effusion with symptoms such as chest pain); pericardial outcome (pericardial effusion with symptoms such as fever or dyspnea); abdominal pain; hepatomegaly; skin rash; subcutaneous mass; headache; epilepsy; vision impairment; motor loss; and diarrhea. The frequency of each outcome was estimated by its relative occurrence in the respective group.

Another pooling was made with regard to the exact Paragonimus species. For Chinese articles that did not report detailed species information, the exact species was assigned according to the geographic location of the cases, if applicable. Following this rationale, which is based on the authors' experience about the distribution of paragonimiasis species in China, cases from the Chinese provinces/municipalities Zhejiang, Shanghai, Jiangsu, Anhui, Jiangxi, Fujian, Jilin, Liaoning, and Beijing were classified as $P$. westermani, whereas cases from the provinces Shaanxi, Sichuan, Hubei, Chongqing, Guilin, and Henan were classified as P. skrjabini. Cases from countries such as Japan and the US remained in the 'not further specified' group.

Disability weights for outcomes (see Table 2) were retrieved in the following order of priority: from the GBD study, i.e. GBD 2013 [16], GBD 2004 [13], and finally - if not available from the previous sources - from other literature.

\section{Decision tree modelling Decision tree}

A decision tree was used to systematically combine the large set of outcomes and their associated DWs with the corresponding frequencies to ultimately estimate summary DWs. The additive approach (Formula 1) was better in combining outcomes that presented separately in individual patients, while the multiplicative approach 


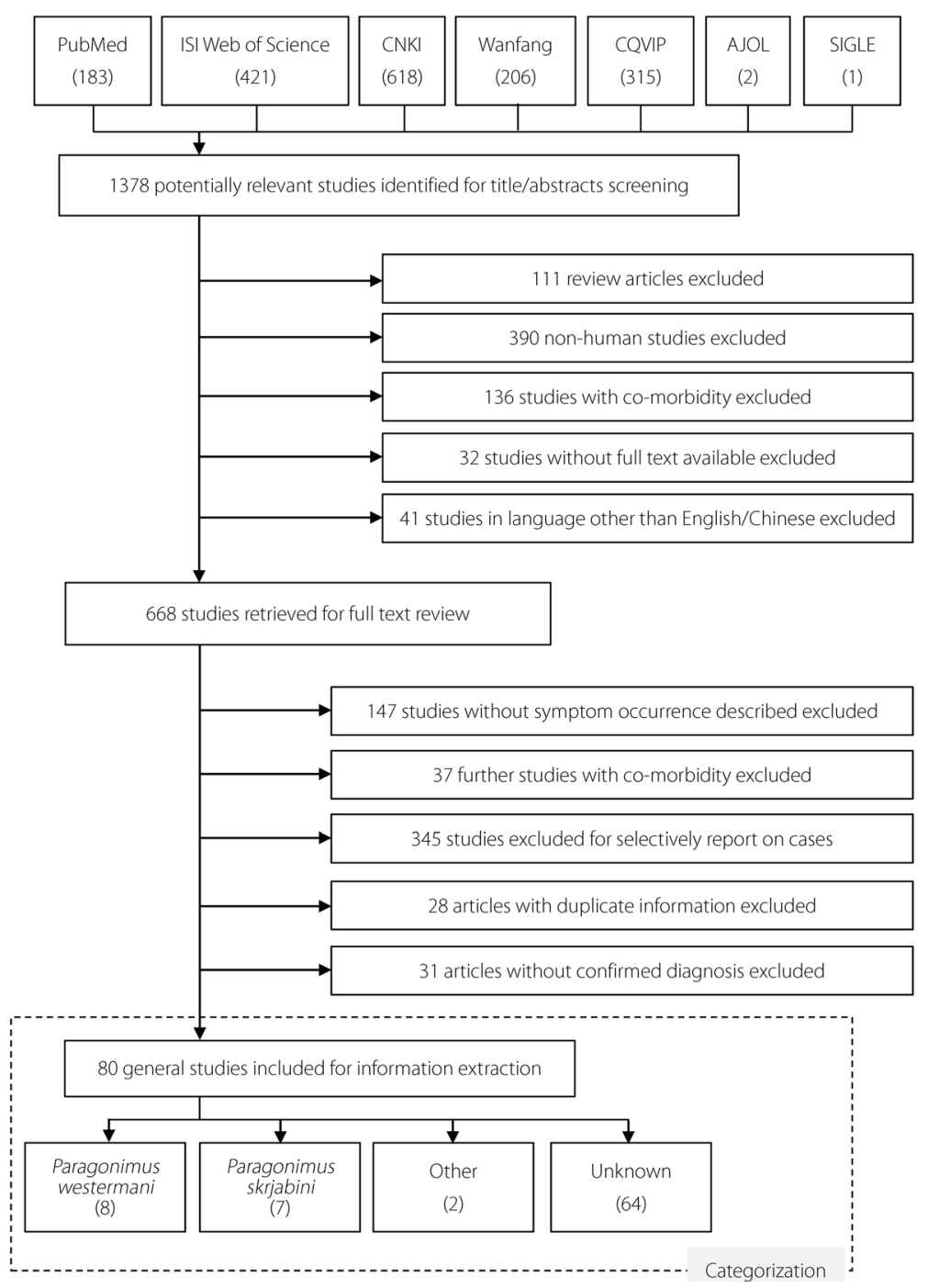

Fig. 1 Article selection flow chart. Figure 1 provides an illustration of the process of how articles were recruited

Table 2 DWs for various outcomes

\begin{tabular}{lllll}
\hline Outcome & DW mean & DW uncertainty level & Source item & Reference \\
\hline Lung outcomes & 0.279 & - & Lower respiratory infection episodes & GBD 2004 \\
Pleural outcomes & 0.054 & $0.035-0.079$ & Lower back pain, moderate (proxy for chest pain) & GBD 2013 \\
Pericardial outcomes & 0.252 & - & Inflammatory heart disease & GBD 2004 \\
Headache & 0.441 & $0.294-0.588$ & Headache, migraine & GBD 2013 \\
Epilepsy & 0.552 & $0.375-0.710$ & Epilepsy, severe & GBD 2013 \\
Motor loss & 0.061 & $0.040-0.089$ & Motor impairment, moderate & GBD 2013 \\
Vision impairment & 0.031 & $0.019-0.049$ & Distance vision impairment, moderate & GBD 2013 \\
Diarrhoea & 0.074 & $0.049-0.104$ & Diarrhea, mild & GBD 2013 \\
Abdominal pain & 0.060 & - & Abdominal pain & Finkelstein et al. 2007 [26] \\
Hepatomegaly & 0.060 & - & Mild/moderate hepatomegaly & Finkelstein et al. 2007 [26] \\
Skin rash & 0.068 & - & Oncocerciasis itching & GBD 2004 \\
Subcutaneous mass & 0.023 & - & Cutaneous leishmaniasis & GBD 2004
\end{tabular}


(Formula 2) was more suitable for combining several outcomes occurring in one patient [27]. As both conditions existed, the two approaches were used respectively in the analysis to compare with each other. $\mathrm{P}_{\text {outcome A }}$ represents the probability/frequency of outcome A; DW outcome A represents the DW of outcome A. The total number of outcomes considered is represented by $n$ in Formulas 1 and 2. All calculations were processed using $\mathrm{R}$ software version 3.1.1 (the R Foundation for Statistical Computing, Vienna, Austria).

$$
\begin{aligned}
& \mathrm{DW}=\sum_{A=1}^{n} P_{\text {outcome } A} * D W_{\text {outcome } A} \\
& \mathrm{DW}=1-\sum_{A=1}^{n}\left(1-P_{\text {outcome } A} * D W_{\text {outcome } A}\right)
\end{aligned}
$$

\section{Base case analysis}

First, we calculated the base case DW for each group. Second, to assess the contribution of each outcome to the overall DW, we recalculated the DW for each group supposing the specific outcome is missing. If the DW changed by more than $10 \%$, the outcome was considered critical.

\section{Uncertainty analysis}

For each group, an uncertainty analysis was conducted to explore the range of overall DWs when all formula inputs varied simultaneously. In each simulation, each for-

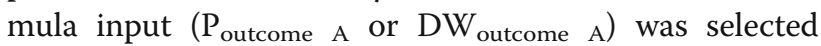
randomly from its specific distribution. The distribution of probability/frequencies followed a beta distribution estimated using the cases in each group. The distribution of DWs followed a logit-normal distribution [28] estimated from the reported mean and range. In total, 5000 Monte Carlo simulations were performed. After summarizing all simulations, we demonstrated the uncertainty interval (UI) by the 2.5 to $97.5 \%$ percentile range of DWs. (For details, see the Additional file 2).

\section{Results}

Among the 80 general articles that were included in this study, 64 did not investigate the species of paragonimiasis. After estimating using cases' locations, 38 articles were categorized in the species group of $P$. westermani and another 38 in the group of P. skrjabini (see Fig. 1 and Table 3).

Table 3 shows the DWs of the different types of paragonimiasis cases. The overall DW estimates of paragonimiasis were 0.1927 (using the additive approach) and 0.1791 (using the multiplicative approach), with 5302 clinical cases from 80 general articles. The DWs estimated using the multiplicative approach were generally lower than those estimated using the additive approach. The DWs of $P$. westermani cases were higher than those of P. skrjabini cases, in both reported and estimated species groups. However, DWs simulated with clinical cases of estimated species showed a smaller gap.

With 5000 Monte Carlo simulations, the findings of the uncertainty analysis showed the magnitude and robustness of DW estimates. The median value of DW estimates in the uncertainty analysis was 0.1956 , with a 95\% UI of $0.1632-0.2378$ using the additive approach, while the value was 0.1816 with a $95 \%$ UI of 0.1530 0.2182 using the multiplicative approach (see Table 3).

Table 4 shows the results of a base case analysis of outcomes. Lung outcomes and headache were the two outcomes that were critical to the overall DW of paragonimiasis, with a greater than $10 \%$ change occurring when the specific outcome was missing from the overall disability weight estimation.

\begin{tabular}{|c|c|c|c|c|c|c|}
\hline \multirow[t]{2}{*}{ Type } & \multirow{2}{*}{$\begin{array}{l}\text { No. of } \\
\text { articles }\end{array}$} & \multirow{2}{*}{$\begin{array}{l}\text { No. of } \\
\text { cases }\end{array}$} & \multicolumn{2}{|c|}{ Additive approach } & \multicolumn{2}{|c|}{ Multiplicative approach } \\
\hline & & & DW & $\begin{array}{l}\text { Uncertainty analysis } \\
\text { [median (2.5-97.5\%)] }\end{array}$ & DW & $\begin{array}{l}\text { Uncertainty analysis } \\
\text { [median (2.5-97.5\%] }\end{array}$ \\
\hline Overall & 80 & 5302 & 0.1927 & $0.1956(0.1632-0.2378)$ & 0.1791 & $0.1816(0.1530-0.2182)$ \\
\hline \multicolumn{7}{|l|}{ Species reported ${ }^{\mathrm{a}}$} \\
\hline P. westermani & 8 & 289 & 0.2829 & $0.2901(0.2274-0.3791)$ & 0.2601 & $0.2660(0.2121-0.3391)$ \\
\hline P. skrjabini & 7 & 701 & 0.0827 & $0.0860(0.0676-0.1115)$ & 0.0804 & $0.0836(0.0659-0.1078)$ \\
\hline Other & 2 & 130 & 0.2073 & $0.2212(0.1646-0.2983)$ & 0.1951 & $0.2067(0.1563-0.2729)$ \\
\hline Unknown & 64 & 4182 & 0.2044 & $0.2077(0.1745-0.2493)$ & 0.1889 & $0.1917(0.1629-0.2272)$ \\
\hline \multicolumn{7}{|c|}{ Species estimation ${ }^{a}$} \\
\hline P. westermani & 38 & 1132 & 0.2464 & $0.2507(0.2067-0.3102)$ & 0.2255 & $0.2291(0.1913-0.2801)$ \\
\hline P. skrjabini & 38 & 3459 & 0.1739 & $0.1770(0.1496-0.2097)$ & 0.1622 & $0.1650(0.1408-0.1934)$ \\
\hline Other & 2 & 581 & 0.2073 & $0.2212(0.1646-0.2983)$ & 0.1951 & $0.2067(0.1563-0.2729)$ \\
\hline Unknown & 3 & 130 & 0.1971 & $0.2014(0.1537-0.2694)$ & 0.1877 & $0.1912(0.1468-0.2543)$ \\
\hline
\end{tabular}

Table 3 Overall paragonimiasis DW and DWs by species

${ }^{a}$ One article reported clinical symptoms of both $P$. westermani and P. skrjabini cases DW Disability weight 
Table 4 Base case analysis of outcomes

\begin{tabular}{lllll}
\hline Outcome & $\begin{array}{l}\text { No. of cases } \\
\text { with the } \\
\text { outcome }\end{array}$ & $\begin{array}{l}\text { Occurrence rate } \\
\text { of cases with } \\
\text { the outcome (\%) }\end{array}$ & Overall DW \% change without the outcome \\
\cline { 3 - 5 } Lung outcomes & 1425 & 26.88 & 38.91 & Additive approach \\
Pleural outcomes & 1341 & 25.29 & 7.09 & 6.35 \\
Pericardial outcomes & 271 & 5.11 & 6.68 & 5.98 \\
Headache & 614 & 11.58 & 26.50 & 24.67 \\
Epilepsy & 90 & 1.70 & 4.86 & 4.34 \\
Motor loss & 86 & 1.62 & 0.51 & 0.45 \\
Vision impairment & 23 & 0.43 & 0.07 & 0.06 \\
Diarrhoea & 268 & 5.05 & 1.94 & 1.72 \\
Abdominal pain & 879 & 16.58 & 5.16 & 4.60 \\
Hepatomegaly & 685 & 12.92 & 4.02 & 3.58 \\
Skin rash & 168 & 3.17 & 1.12 & 0.99 \\
Subcutaneous mass & 1391 & 26.24 & 3.13 & 2.78
\end{tabular}

DW Disability weight

The differences in DW estimates between various types of paragonimiasis were further analysed by comparing outcome contributions to the overall estimates.

Figure 2 shows the comparison of the outcome probability, DW contribution, and its percentage share (using the additive approach) between $P$. westermani cases and
P. skrjabini cases. Lung outcomes and headache were the top two contributors to DW estimates for both species. Compared to P. skrjabini cases, lung outcomes were more probable to occur in $P$. westermani cases and had more percentage share, thus bringing more contributions to the overall DW estimate of the latter species. Subcutaneous

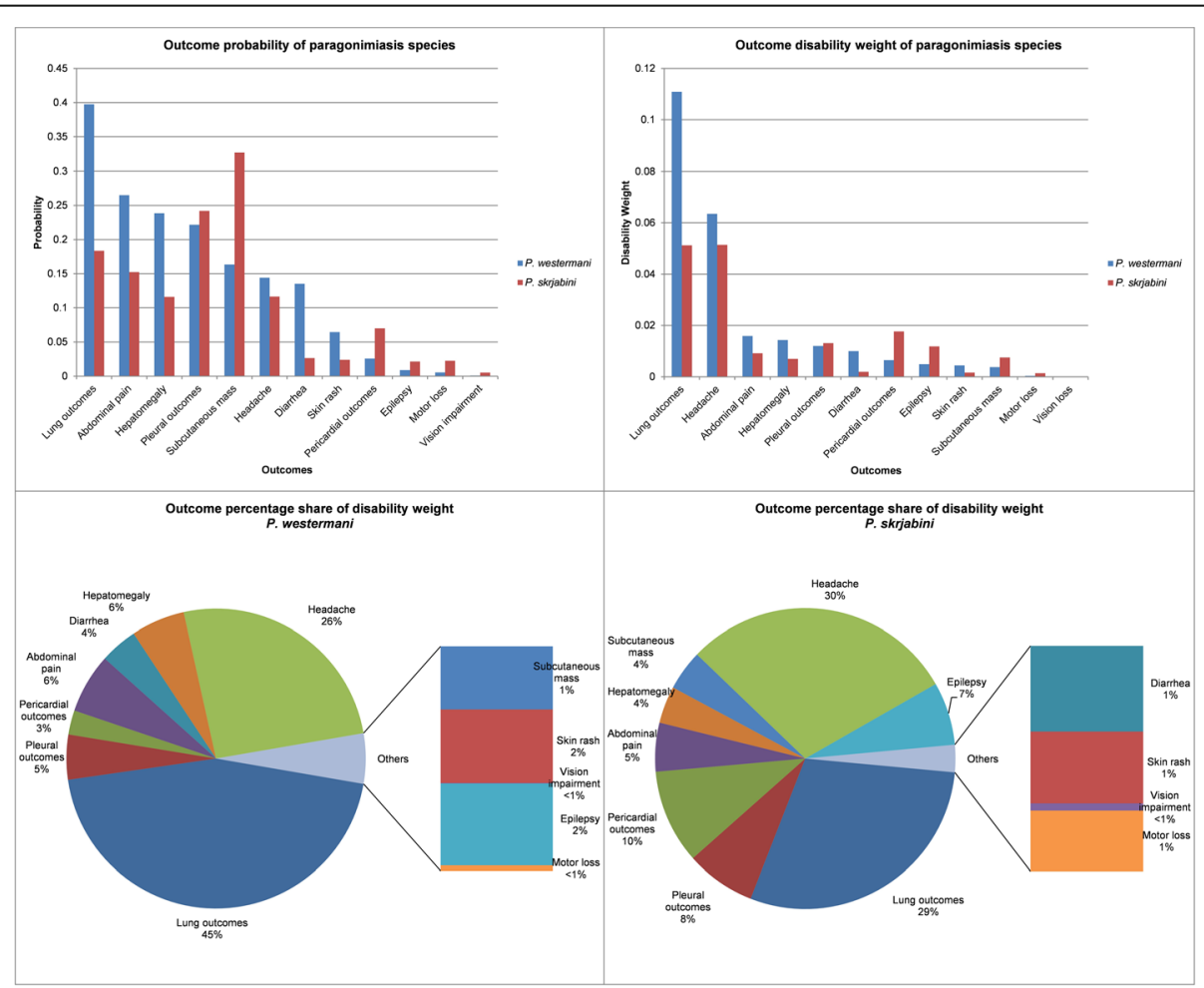

Fig. 2 Outcome comparison by species. Figure 2 shows the comparison of the outcome probability, DW contribution, and its percentage share between Paragonimus westermani cases and P. skrjabini cases. DW: Disability weight 
mass was more likely to occur in P. skrjabini cases, but resulted in less impact to its DW estimates compared with P. westermani cases (see Fig. 2).

\section{Discussion}

Our study simulated the overall DW estimate of paragonimiasis at 0.1927 (median 0.1956) using the additive approach and 0.1791 (median 0.1816) using the multiplicative approach. The estimated DW of $P$. westermani was higher than that of P. skrjabini. Lung outcomes and headache contributed the greatest to DW estimates.

Compared with the GBD 2010 study, this study found considerably higher DWs than the health status of "moderate abdominal pelvic problem" (0.123) that Salomon et al. $[15,28]$ used as a proxy for the outcomes of both heavy paragonimiasis and cerebral paragonimiasis. However, abdominal pelvic problem is never the dominant manifestation even if sometimes abdominal organs could be involved [2].

Our DW estimates are also higher than those Fürst et al. [3] used for heavy paragonimiasis (0.099) and cerebral paragonimiasis $(0.100)$. The reason could be that we included more outcomes in our model, such as headache and pericardial symptoms. The necessity of these extra outcomes was manifested by their non-negligible contribution to the overall DW. However, our DW estimates were lower than either the heavy paragonimiasis or cerebral paragonimiasis DWs used in GBD 2013 and GBD 2016 [16-18], as they focus on more severe situation with higher worm burden or cerebral involvement. The application of the two different types of DWs in the years lived with disability (YLD) calculation shall have to match with different incidence/prevalence rates. However, it was often difficult to find the morbidity information relating to heavy/cerebral paragonimiasis for a specific region in the literature search [18]. Therefore, the overall paragonimiasis DW estimated in this study that match just overall incidence/prevalence rate in YLD calculation could better fit the data paucity situation.

Our result is higher than the health status DW pertaining to acute episode of severe infectious disease (0.133) and a bit lower than that of post-acute effects (fatigue, emotional lability, and insomnia) of infectious diseases (0.219) as outlined in GBD 2013 [16, 24]. Compared with Clonorchis sinensis, another foodborne trematode disease, our estimate is higher than the DW simulated by Qian et al. [29] using data collected from a field survey. Compared with the DW Finkelstein et al. [26] estimated for schistosomiasis using a decision tree (0.132), our result is also higher. Apart from the difference between diseases, the reason could be partly due to data sources. Most of our recruited articles were case reviews based on clinical cases that referred to hospitals as field surveys were either unavailable or reported insufficient information. This may be associated with the overestimation of the severity of a disease.

We further found that headache contributed more to the DW estimation than epilepsy. Headache is probably a neglected outcome for cerebral paragonimiasis in disease burden estimation. None of the GBD studies [3, 15, $18,30]$ considered headache as an important outcome of cerebral paragonimiasis. In a FERG estimation to compare DALYs of foodborne diseases, the DW of paragonimiasis also just considered the epilepsy outcome of cerebral symptoms [21]. Although paragonimiasis DALY ranked high in a comparison of foodborne diseases [22], it could still be underestimated with the neglect of headache impact to the DW used in calculation.

Our study generated DWs estimated using the additive approach and the multiplicative approach. The result showed that the DW estimated using the additive approach was generally higher than that estimated using the multiplicative approach. The limitation of the additive approach could be one of the reasons. Using this approach, the combined DW could exceed 1.0 and overestimation could occur when different outcomes affect the same health domain [27] (e.g. the lung outcome and the pericardial outcome could both lead to symptoms in the chest).

The DWs of $P$. westermani were higher than that of P. skrjabini for both reported and estimated species. For the reported species, their simulated 95\% UIs did not overlap, while for the estimated species, their simulated 95\% UIs overlapped for both the additive approach and the multiplicative approach. As the overlapped part was relatively small and the species was estimated based on the authors' experiences, we still consider the difference to be somewhat significant. It was inferred that a possible reason could be the biological difference between the two species. $P$. westermani larvae develop into adult worms in the human lung, while only a few P. skrjabini larvae develop into adults in the same location. Most of the latter remain in the juvenile stage, migrating to different organs and causing damage [10]. Therefore, the analysis of outcome percentage share of the estimated DWs further showed that compared with $P$. westermani, the estimated P. skrjabini cases had higher percentage of extra-pulmonary outcomes.

In our study, only two articles with the species $P$. africanus and P. miyazaki were included in the modelling. The simulated DW was in between those of $P$. westermani and P. skrjabini. It was further inferred that some other species might be similar to $P$. westermani in DW estimates as they mainly cause pulmonary symptoms, including $P$. africanus, $P$. heterotremus, $P$. uterobilateralis, $P$. kellicotti, and P. mexicanus. These species distribute across West Africa, South and Southeast Asia, and North and South America [2]. Some other species could be similar to P. skrjabini 
with ectopic forms more common, such as $P$. miyazaki from Japan, which is very close morphologically and molecularly to P. skrjabini from China [2].

We recognize that our study had some limitations. We excluded case reviews in languages other than Chinese and English due to article availability and language barriers. We missed articles with full text non-available to us. This may bring bias to our study, however, we tried to minimize impact as most of the missed 32 articles were published earlier than in 1995 . We also omitted some scarce outcomes such as spinal symptoms and eye symptoms, which may lead to an underestimation of the overall DW. In the uncertainty analysis, we replaced the missing range of some outcome DWs with the average value of available uncertainty levels. This may result in our uncertainty results having lower credibility. In addition, as most Chinese cases did not report species information, we estimated the species based on authors' experiences on species information collected from cases in the same location. This kind of estimation may reduce the solidity of species classification.

Despite these potential limitations, our estimation of the DW of paragonimiasis helps to close the gap in the current disease burden system, particularly for NTDs. In low- and middle-income countries, such as China, paragonimiasis is increasingly becoming a potential threat, as the production and consumption of crayfish, the second intermediate host, has greatly risen in recent years. The national production of crayfish increased by $36.59 \%$ in 2017 compared with 2016, and for some provinces, the rate was as high as $141.05 \%$; meanwhile, the number of crayfish-specific restaurants boomed in major cities like Shanghai and Guangzhou [31]. We call for more attention on this chronic disabling and death threatening "poverty disease" [32]. More studies are needed in areas related to paragonimiasis assessment, prevention, and treatment.

\section{Conclusions}

Our study systematically estimated the DWs of paragonimiasis from reported cases for the first time. P. westermani was found to have higher DW estimates than $P$. skrjabini. These could bring considerable changes to disease burden estimation, thus having an impact on health policymaking and resource allocation in research, monitoring, and control, particularly when faced with the potentially increasing threat of paragonimiasis in low- and middle-income countries.

\section{Additional file}

Additional file 1: Multilingual abstracts in the five official working languages of the United Nations. (PDF $225 \mathrm{~kb}$ )

Additional file 2: Data modelling details. (DOCX $26 \mathrm{~kb}$ )

\section{Abbreviations}

COPD: Chronic obstructive pulmonary disease; DALY: Disability-adjusted life year; DW: Disability weight; FERG: Foodborne Disease Burden Epidemiology Reference Group; GBD: Global burden of disease; HIV: Human

immunodeficiency virus; NTD: Neglected tropical disease; TB: Tuberculosis; UI: Uncertainty interval; WHO: World Health Organization; YLD: Years lived with disability

\section{Funding}

This study is supported by the International Development Research Centre (IDRC), Canada (grant no. 105509-00001002-024); Public Health Research Center, Jiangnan University (grant no. BM2015024); Outstanding Youth Fund, Jiangsu Institute of Parasitic Diseases; Jiangsu Provincial Project of Invigorating Health Care through Science, Technology and Education; and in part by a grant from the National Nature Science Foundation (grant no. 81573261) and the Health Promotion Project, Outstanding Person Fund, Jiangsu Provincial Department of Health (2011).

\section{Availability of data and materials}

The datasets used and/or analysed during the current study are available from the corresponding author upon reasonable request.

\section{Authors' contributions}

YF designed the study, collected, and analysed the data on paragonimiasis cases, and wrote the paper. TF was a major contributor in improving the study design and paper. LL contributed to the improvement of data collection and analysis. G-JY was a major contributor to study design, data analysis, and improving the paper. All authors read and approved the final paper.

Ethics approval and consent to participate

Not applicable.

\section{Consent for publication \\ Not applicable.}

\section{Competing interests}

The authors declare that they have no competing interests.

\section{Author details \\ ${ }^{1}$ Jiangsu Institute of Parasitic Diseases, 117 Meiyuan Yangxiang, Wuxi City, Jiangsu Province 214064, People's Republic of China. ${ }^{2}$ Key Laboratory of National Health and Family Planning Commission on Parasitic Disease Control and Prevention, Wuxi, People's Republic of China. ${ }^{3}$ Jiangsu Provincial Key Laboratory on Parasite and Vector Control Technology, Wuxi, People's Republic of China. ${ }^{4}$ Department of Epidemiology and Public Health, Swiss Tropical and Public Health Institute, Socinstrasse 57, CH-4002 Basel, Switzerland. ${ }^{5}$ University of Basel, Basel, Switzerland.}

Received: 2 March 2018 Accepted: 12 September 2018

Published online: 19 October 2018

\section{References}

1. WHO. Working to overcome the global impact of neglected tropical diseases: first WHO report on neglected tropical diseases, 2010. In: Neglected tropical diseases. WHO. 2010. http://www.who.int/neglected_ diseases/2010report/en/. Accessed 19 Jan 2018.

2. Blair D. Paragonimiasis. Adv Exp Med Biol. 2014;766:115-52.

3. Fürst T, Keiser J, Utzinger J. Global burden of human food-borne trematodiasis: a systematic review and meta-analysis. Lancet Infect Dis. 2012;12(3):210-21.

4. Fürst T, Duthaler U, Sripa B, Utzinger J, Keiser J. Trematode infections: liver and lung flukes. Infect Dis Clin North Am. 2012;26(2):399-419.

5. Devi KR, Narain K, Bhattacharya S, Negmu K, Agatsuma T, Blair D, et al. Pleuropulmonary paragonimiasis due to Paragonimus heterotremus: molecular diagnosis, prevalence of infection and clinicoradiological features in an endemic area of northeastern India. Trans R Soc Trop Med Hyg. 2007; 101(8):786-92

6. Nawa Y, Hatz C, Blum J. Sushi delights and parasites: the risk of fishborne and foodborne parasitic zoonoses in Asia. Clin Infect Dis. 2005:41(9):1297-303. 
7. FAO. FAO Yearbook. Fishery and aquaculture statistics 2010-2016. In: Publications. Rome: FAO of the United Nations. p. 2017. http://www.fao. org//fishery/publications/yearbooks/en. Accessed 19 Jan 2018.

8. Keiser J, Utzinger J. Emerging foodborne trematodiasis. Emerg Infect Dis. 2005;11(10):1507-14.

9. Fürst T, Sayasone S, Odermatt P, Keiser J, Utzinger J. Manifestation, diagnosis, and management of foodborne trematodiasis. BMJ. 2012;344: e4093.

10. Liu Q, Wei F, Liu W, Yang S, Zhang X. Paragonimiasis: an important foodborne zoonosis in China. Trends Parasitol. 2008;24(7):318-23.

11. Keiser J, Utzinger J. Food-borne trematodiases. Clin Microbiol Rev. 2009; 22(3):466-83.

12. Yang JS, Chen MG, Zheng F, Blair D. Paragonimus and paragomimiasis in China: a review of the literature. Chin J Parasitol Parasit Dis. 2000(Special Issue):1-78 (in Chinese).

13. WHO. Global burden of disease 2004 Update: disability weights for diseases and conditions. In: Health statistics and information systems. WHO, 2004. http://www.who.int/healthinfo/global_burden_disease/GBD2004_ DisabilityWeights.pdf. Accessed 19 Jan 2018.

14. Murray CJ, Ezzati M, Flaxman AD, Lim S, Lozano R, Michaud C, et al. GBD 2010: design, definitions, and metrics. Lancet. 2012;380(9859):2063-6.

15. Salomon JA, Vos T, Hogan DR, Gagnon M, Naghavi M, Mokdad A, et al. Common values in assessing health outcomes from disease and injury: disability weights measurement study for the global burden of disease study 2010. Lancet. 2012;380(9859):2129-43.

16. Salomon JAHJ, Davis A, Maetens de Noordhout C, Polinder S, Havelaar AH, Cassini A, Devleesschauwer B, Kretzschmar M, Speybroeck N, Murray CJL, Vos T. Disability weights for the global burden of disease 2013 study. Lancet Glob Health. 2015;3:e712-23.

17. Global Burden of Disease Study 2013 Collaborators. Global, regional, and national incidence, prevalence, and years lived with disability for 301 acute and chronic diseases and injuries in 188 countries, 1990-2013: a systematic analysis for the Global Burden of Disease Study 2013. Lancet. 2015; 386(9995):743-800.

18. GBD 2016 Disease and Injury Incidence and Prevalence Collaborators. Global, regional, and national incidence, prevalence, and years lived with disability for 328 diseases and injuries for 195 countries, 1990-2016: a systematic analysis for the Global Burden of Disease Study 2016. Lancet. 2017;390(10100):1211-59.

19. Blair D, Agatsuma T, Wang W. Paragonimiasis. In: Murrell KD, Fried B, editors World Class Parasites, Vol 11. World Class Parasites Dordrecht. Netherlands: Springer; 2007. p. 117-50.

20. Torgerson PR, Devleesschauwer B, Praet N, Speybroeck N, Willingham AL, Kasuga F, et al. World Health Organization estimates of the global and regional disease burden of 11 foodborne parasitic diseases, 2010: a data synthesis. PLoS Med. 2015;12(12):e1001920.

21. Devleesschauwer B, Haagsma JA, Angulo FJ, Bellinger DC, Cole D, Dopfer D, et al. Methodological framework for World Health Organization estimates of the global burden of foodborne disease. PLoS One. 2015. https://doi.org/10. 1371/journal.pone.0142498.

22. Havelaar AH, Kirk MD, Torgerson PR, Gibb HJ, Hald T, Lake RJ, et al. World Health Organization Global Estimates and Regional Comparisons of the Burden of Foodborne Disease in 2010. PLoS medicine. 2015. https://doi.org/ 10.1371/journal.pmed.1001923.

23. Murray CJ, Vos T, Lozano R, Naghavi M, Flaxman AD, Michaud C, et al. Disability-adjusted life years (DALYS) for 291 diseases and injuries in 21 regions, 1990-2010: a systematic analysis for the Global Burden of Disease Study 2010. Lancet. 2012;380(9859):2197-223.

24. Murray CJL, Ezzati M, Flaxman A. Supplementary appendix - Comprehensive Systematic Analysis of Global Epidemiology : Definitions, Methods, Simplification of DALYs, and Comparative Results from the Global Burden of Disease Study 2010. Lancet. 2012;380:2063-6.

25. GBD 2013 DALYs and HALE Collaborators. Global, regional, and national disability-adjusted life years (DALYs) for 306 diseases and injuries and healthy life expectancy (HALE) for 188 countries, 1990-2013: quantifying the epidemiological transition. Lancet. 2015;386(10009):2145-91.

26. Finkelstein $\mathrm{J}$, Schleinitz MD, Carabin H, McGarvey ST. Decision-model estimation of the age-specific disability weight for schistosomiasis japonica: a systematic review of the literature. PLoS neglected tropical diseases. 2008. https://doi.org/10.1371/journal.pntd.0000158.
27. Haagsma JA, van Beeck EF, Polinder S, Toet H, Panneman M, Bonsel GJ. The effect of comorbidity on health-related quality of life for injury patients in the first year following injury: comparison of three comorbidity adjustment approaches. Population health metrics. 2011;9:10.

28. Salomon JAMC, Ustun TB, Chatterji S. Health state valuations in summary measures of population health. In: Murray CJLED, editor. Health systems performance assessment: debate, methods, and empiricism. Geneva: World Health Organization; 2003. p. 409-36.

29. Qian MB, Chen YD, Fang YY, Xu LQ, Zhu TJ, Tan T, et al. Disability weight of Clonorchis sinensis infection: captured from community study and model simulation. PLoS neglected tropical diseases. 2011. https://doi.org/10.1371/ journal.pntd.0001377.

30. Global Burden of Disease Study 2013 Collaborators. Global, regional, and national incidence, prevalence, and years lived with disability for 301 acute and chronic diseases and injuries in 188 countries, 1990-2013: a systematic analysis for the Global Burden of Disease Study 2013. Lancet. 2015; 386(9995):743-800.

31. China National Fisheries Managing Beaurau, National Fisheries Technology Extension Center, China Society of Fisheries. China Crayfish Industry Development Report (2018). In: Work dynamics: China Society of Fisheries; 2018. http://www.nftec.agri.cn/gzdt/201806/t20180615_6173297.htm. Accessed 15 Aug 2018 (in Chinese).

32. Zhou XN. Status and future focus of the national control program on parasitic diseases. Chin J Schisto Control. 2011:23(05):473-5 (in Chinese).

\section{Ready to submit your research? Choose BMC and benefit from:}

- fast, convenient online submission

- thorough peer review by experienced researchers in your field

- rapid publication on acceptance

- support for research data, including large and complex data types

- gold Open Access which fosters wider collaboration and increased citations

- maximum visibility for your research: over $100 \mathrm{M}$ website views per year

At $\mathrm{BMC}$, research is always in progress.

Learn more biomedcentral.com/submissions 